\title{
RESEARCH
}

Open Access

\section{Prognostic accuracy of SOFA, qSOFA and SIRS criteria in hematological cancer patients: a retrospective multicenter study}

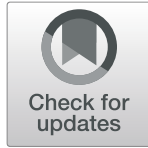

Lucie Probst ${ }^{1}$, Enrico Schalk ${ }^{2}$, Tobias Liebregts ${ }^{3}$, Vanja Zeremski ${ }^{2}$, Asterios Tzalavras ${ }^{3}$,

Michael von Bergwelt-Baildon ${ }^{4}$, Nina Hesse ${ }^{1}$, Johanna Prinz ${ }^{1}$, Jörg Janne Vehreschild ${ }^{1,5,6}$,

Alexander Shimabukuro-Vornhagen ${ }^{1}$, Dennis A. Eichenauer ${ }^{1}$, Jorge Garcia Borrega', Matthias Kochanek',

Boris Böll ${ }^{1^{*}}$ and for the Working Party on Intensive Care Medicine in Hematologic and Oncologic Patients

(iCHOP) of the German Society of Hematology and Medical Oncology (DGHO)

\begin{abstract}
Background: With Sepsis-3, the increase in sequential organ failure assessment (SOFA) as a clinical score for the identification of patients with sepsis and quickSOFA (qSOFA) for the identification of patients at risk of sepsis outside the intensive care unit (ICU) were introduced in 2016. However, their validity has been questioned, and their applicability in different settings and subgroups, such as hematological cancer patients, remains unclear. We therefore assessed the validity of SOFA, qSOFA, and the systemic inflammatory response syndrome (SIRS) criteria regarding the diagnosis of sepsis and the prediction of in-hospital mortality in a multicenter cohort of hematological cancer patients treated on ICU and non-ICU settings.
\end{abstract}

Methods: We retrospectively calculated SIRS, SOFA, and qSOFA scores in our cohort and applied the definition of sepsis as "life-threatening organ dysfunction caused by dysregulated host response to infection" as reference. Discriminatory capacity was assessed using the area under the receiver operating characteristic curve (AUROC).

Results: Among 450 patients with hematological cancer (median age 58 years, 274 males [61\%]), 180 (40\%) had sepsis of which 101 (56\%) were treated on ICU. For the diagnosis of sepsis, sensitivity was 86\%, 64\%, and 42\% for SIRS, SOFA, and QSOFA, respectively. However, the AUROCs of SOFA and qSOFA indicated better discrimination for sepsis than SIRS (SOFA, 0.69 [95\% Cl, 0.64-0.73] $p<0.001$; qSOFA, 0.67 [95\% Cl, 0.62-0.71] $p<0.001 ;$ SIRS, 0.57 [95\% $\mathrm{Cl}, 0.53-0.61] p<0.001)$. In-hospital mortality was $40 \%$ and $14 \%$ in patients with and without sepsis, respectively $(p<0.001)$. Regarding patients with sepsis, mortality was similar in patients with positive and negative SIRS scores (39\% vs. 40\% ( $p=0.899$ ), respectively). For patients with qSOFA $\geq 2$, mortality was $49 \%$ compared to $33 \%$ for those with qSOFA $<2(p=0.056)$, and for SOFA $56 \%$ vs. $11 \%(p<0.001)$, respectively. SOFA allowed significantly better discrimination for in-hospital mortality (AUROC 0.74 [95\% Cl, 0.69-0.79] $p<0.001$ ) than qSOFA (AUROC $0.65[95 \% \mathrm{Cl}, 0.60-0.71] p<0.001$ ) or SIRS (AUROC 0.49 [95\% Cl, 0.44-0.54] $p<0.001$ ).

(Continued on next page)

\footnotetext{
* Correspondence: boris.boell@uk-koeln.de

${ }^{1}$ University of Cologne, Department I of Internal Medicine, Center for

Integrated Oncology Aachen Bonn Cologne Duesseldorf, Cologne, Germany

Full list of author information is available at the end of the article
}

(c) The Author(s). 2019 Open Access This article is distributed under the terms of the Creative Commons Attribution 4.0 International License (http://creativecommons.org/licenses/by/4.0/), which permits unrestricted use, distribution, and reproduction in any medium, provided you give appropriate credit to the original author(s) and the source, provide a link to the Creative Commons license, and indicate if changes were made. The Creative Commons Public Domain Dedication waiver (http://creativecommons.org/publicdomain/zero/1.0/) applies to the data made available in this article, unless otherwise stated. 
(Continued from previous page)

Conclusions: An increase in SOFA score of $\geq 2$ had better prognostic accuracy for both diagnosis of sepsis and in-hospital mortality in this setting, and especially on ICU, we observed limited validity of SIRS criteria and qSOFA in identifying hematological patients with sepsis and at high risk of death.

Keywords: Sepsis, SIRS, Sepsis-3, qSOFA, Cancer, Hematological malignancies

\section{Background}

Sepsis is among the leading causes of death with an estimated annual global incidence of 31.5 million cases of sepsis and 19.4 million cases of severe sepsis [1]. Although outcomes have improved due to collaborative efforts [2], mortality remains high with estimated in-hospital mortality rates of $17-26 \%$. In patients presenting with septic shock, the estimated mortality is even higher with rates of $46 \%$ and higher depending on the definition [3].

Accurate definition, diagnosis, and early recognition of sepsis are crucial for the effective management of patients, as it improves outcomes [4, 5]. Sepsis and septic shock were redefined in 2016 (Sepsis-3), defining sepsis as "lifethreatening organ dysfunction caused by a dysregulated host response to infection" [6]. An increase in the sequential organ failure assessment score (SOFA) of $\geq 2$ was associated with an in-hospital mortality greater than $10 \%$ and proposed for clinical operationalization [6].

As a bedside screening tool for sepsis-related mortality for non-intensive care settings, the quickSOFA (qSOFA) score was introduced to rapidly identify patients with high likelihood of poor sepsis-related outcome, in particular with high risk of sepsis-related mortality [3, 6, 7]. Although, the effort of establishing a new definition and clinical criteria by analyzing large datasets of patient records was widely acknowledged, the validity of these criteria for clinical practice has been questioned [8-12] and their applicability in different settings and subgroups remains unclear [13, 14]. This might particularly apply to specific subgroups such as burn patients [15], surgical patients [16], and hematological cancer patients $[14,17]$.

Cancer patients and particularly patients with hematological malignancies commonly suffer from immunodeficiency resulting from the underlying disease and/or from immunosuppressive therapy and are therefore at high risk of infection resulting in sepsis $[18,19]$. Importantly, population and register-based studies indicate that up to one in five patients admitted to intensive care units (ICU) suffer from underlying malignancies and that sepsis is the leading cause for ICU admission in patients with hematologic malignancies [18, 20-22], although the validity of sepsis criteria in hematological cancer patients remains unclear.
We therefore analyzed the discriminative accuracy of the Sepsis-3 scores and the systemic inflammatory response syndrome (SIRS) criteria for sepsis and in-hospital mortality in a multicenter cohort of hematological cancer patients. The primary aim of this study was to assess the impact of different sepsis scores in discriminating sepsis and in-hospital mortality and to validate their use in these patients admitted to ICU or a regular hematology ward.

\section{Methods}

This was a retrospective, multicenter study of 450 adult patients ( $\geq 18$ years) with hematological cancer admitted between 2013 and 2018 to a regular ward specialized in hematology or the ICU at one of the following three large academic tertiary care hospitals in Germany (University Hospital of Cologne, University Hospital Magdeburg, University Hospital Essen). The patients were admitted for application of therapy or for treatment of complications consecutive to their underlying disease or therapy such as fever and suspected infection. For the ICU patients included in the study, patients with hematological cancer consecutively admitted to an ICU at one of the three centers were included in the study irrespective of the suspected diagnosis at transfer to the ICU. Patients on the normal hematology ward of one of the three centers were included, when an infection was suspected, suspected infection being defined according to current Surviving Sepsis Campaign guidelines and recommendations (administration of antibiotics excluding prophylactic treatment and sampling of body fluid for culturing) [2, 6]. For patients with multiple hospitalizations, we only included their first admission.

The following clinical data were taken from patients' medical records documented at admission to ICU or when an infection was suspected: patient age, gender, underlying disease and current status of the malignancy (newly diagnosed, partial or complete remission, stable disease, progressive disease), data on hematopoietic stem cell transplant (HSCT) and source of infection, physiological and laboratory data to determine the sepsis scores such as the SIRS criteria [23], the SOFA and the qSOFA score [6], severity indicators such as the Acute Physiology, Age, Chronic Health Evaluation II (APACHE II) score [24], Glasgow Coma Scale (GCS) [25], acute kidney failure and 
graft-versus-host disease, measures of outcome such as in-hospital mortality, ICU mortality, hospital length of stay and transfer to ICU. Additionally, lactate levels, administration of vasopressors, and mean arterial pressure (MAP) were collected to determine septic shock according to the Sepsis-3 definition from 2016 [3].

According to current Surviving Sepsis Campaign guidelines and recommendations [2, 6], all patients who received antibiotics (excluding prophylactic treatment) and underwent sampling of body fluids for culturing were defined as patients with suspected infection [6], and patients with "life-threatening organ dysfunction caused by a dysregulated host response to infection" were defined as patients with sepsis [6]. For each scoring system, the standard threshold of $\geq 2$ points was applied for a positive SIRS, SOFA, and qSOFA score $[6,26]$. The baseline SOFA score to differentiate between preexisting and acute changes in organ dysfunction in order to calculate the acute increase in SOFA was assessed with the help of prior laboratory values, the impact of the underlying disease and its therapies. In case of missing data to determine the SIRS, SOFA, and qSOFA score, the patients were excluded from further analysis of diagnostic accuracy. Patients with sepsis and a vasopressor requirement to maintain a MAP of $\geq 65 \mathrm{mmHg}$ and a serum lactate level $>2 \mathrm{mmol} / \mathrm{L}$ in the absence of hypovolemia were diagnosed with septic shock according to the Sepsis-3 definition [6].

\section{Statistical analysis}

Dichotomous variables were presented as absolute numbers and percentages and continuous variables as medians and interquartile ranges (IQR). The MannWhitney $U$ test was used to compare continuous variables between the two groups "patients with sepsis" and "patients without sepsis" after testing for normal distribution using the Shapiro-Wilk test. Categorical binary variables were compared by the chi-squared test. Two-sided $p$ values $<0.05$ were required for statistical significance.

By applying the definition of sepsis as "life-threatening organ dysfunction caused by a dysregulated host response to infection" [6] as reference, the scores were evaluated. In order to assess the accuracy of the three investigated sepsis criteria, SIRS, SOFA, and qSOFA, regarding sepsis and in-hospital mortality, sensitivity, specificity, predictive values, likelihood ratios (LR), and the Youden's Index were calculated according to standard formulas as previously described, and the area under receiver operating characteristic (AUROC) curves were determined [27-30]. Pairwise comparison of receiver operating characteristic (ROC) curves was conducted in order to test statistical significance. To further assess the association between sepsis scoring systems and mortality, we also determined the in-hospital mortality for patients with positive and negative sepsis scores and calculated the risk ratio (RR) for each sepsis criterion. All statistical analyses were two-tailed and calculated with Stata/SE 13.

\section{Results \\ Study population}

A total of 450 patients with hematological malignancies were included from ICUs or regular hematology wards of three German tertiary academic hospitals. The median age was 58 years (IQR, 46-66 years), 61\% were male, $44 \%$ had leukemia, $31 \%$ had lymphoma, $18 \%$ suffered from myeloma, and $4 \%$ from myelodysplastic syndrome. Nearly half the population was in partial or complete remission, about one in four patients were newly diagnosed and about one in five patients suffered from disease progression at admission. About $40 \%$ had received a HSCT, of which half the patients an allogeneic (49\%; Table 1).

One hundred eighty patients (40\%) presented with sepsis of which 101 (56\%) were treated on ICU and an additional 27 patients (15\%) were later transferred to an ICU (Table 2). The two subgroups of patients (sepsis vs. no sepsis) had a similar distribution regarding age and gender but differed significantly in platelet count, creatinine and bilirubin. Patients with sepsis were more likely to present with acute kidney failure, lower MAP, and higher heartrates at admission. The measured body temperature was not significantly different between the two groups. The most common source of infection in patients with sepsis was the respiratory tract (32\%) followed by central line-associated infections and the gastrointestinal tract both in $9 \%$ of the patients (Tables 1 and 2).

\section{Performance of SIRS, SOFA and qSOFA criteria}

Within the group of patients with sepsis, $86 \%$ (95\% confidence interval $[95 \% \mathrm{CI}], 80-90 \%)$ of the patients presented with a $\geq 2$ positive SIRS criteria, $64 \%$ (95\% CI, $57-71 \%)$ of the patients with a SOFA score $\geq 2,42 \%$ (95\% CI, 34-49\%) of the patients with a qSOFA score $\geq$ 2 , and 20 patients (12\%) fulfilled the Sepsis-3 criteria of septic shock. Thus, the false-negative rate for the SIRS criteria was $14 \%$, for the SOFA score $36 \%$, and for the qSOFA score $59 \%$. Within the group of patients not suffering from sepsis, $76 \%$ of the patients presented with $\geq$ 2 SIRS criteria, $26 \%$ with a SOFA score $\geq 2$, and $9 \%$ with a qSOFA score $\geq 2$, representing the number of false positives for each score, respectively (Table $2,2 \times 2$ contingency tables and flow charts for each score are provided in Additional files 1 and 2). 
Table 1 Demographics and clinical characteristics of the cohort

\begin{tabular}{|c|c|c|c|c|}
\hline Variable & Total cohort $(n=450)$ & Patients with sepsis $(n=180)$ & Patients without sepsis $(n=270)$ & $p$ value \\
\hline \multicolumn{5}{|l|}{ Demographics } \\
\hline Age in years, median (IQR) & $58(46-66)$ & $58(44.5-66)$ & $59(46-66)$ & 0.876 \\
\hline Male gender & $274(60.9)$ & $106(58.9)$ & $168(62.2)$ & 0.478 \\
\hline Underlying malignancy & & & & 0.177 \\
\hline Non-Hodgkin's lymphoma & $113(25.1)$ & $39(21.7)$ & $74(27.4)$ & \\
\hline Hodgkin lymphoma & $25(5.6)$ & $11(6.1)$ & $14(5.2)$ & \\
\hline Acute myeloid leukemia & $127(28.2)$ & $52(28.9)$ & $75(27.8)$ & \\
\hline Acute lymphocytic leukemia & $36(8.0)$ & $15(8.3)$ & $21(7.8)$ & \\
\hline Multiple myeloma & $80(17.8)$ & $30(16.7)$ & $50(18.5)$ & \\
\hline Chronic myeloid leukemia & $9(2.0)$ & $6(3.3)$ & $3(1.1)$ & \\
\hline Chronic lymphocytic leukemia & $25(5.6)$ & $8(4.4)$ & $17(6.3)$ & \\
\hline Myelodysplastic syndrome & $18(4.0)$ & $10(5.6)$ & $8(3.0)$ & \\
\hline Other & $17(3.8)$ & $9(5.0)$ & $8(3.0)$ & \\
\hline Disease status at admission & & & & 0.184 \\
\hline Newly diagnosed & $118(26.2)$ & $40(22.2)$ & $78(28.9)$ & \\
\hline Partial or complete remission & $202(44.9)$ & $86(47.8)$ & $116(43.0)$ & \\
\hline Stable disease & $14(3.1)$ & $5(2.8)$ & $9(3.3)$ & \\
\hline Progressive disease & $91(20.2)$ & $34(18.9)$ & $57(21.1)$ & \\
\hline Unknown & $25(5.6)$ & $15(8.3)$ & $10(3.7)$ & \\
\hline Hematopoietic stem cell transplant & $184(40.9)$ & $82(45.6)$ & $102(37.8)$ & 0.100 \\
\hline Allogeneic & $87(47.3)$ & $45(54.9)$ & $42(41.2)$ & \\
\hline Autologous & $94(51.1)$ & $36(43.9)$ & $58(56.9)$ & \\
\hline \multicolumn{5}{|l|}{ Infection site (patients with sepsis) } \\
\hline Respiratory tract & & $58(32.2)$ & & \\
\hline Central line-related & & $17(9.4)$ & & \\
\hline Gastrointestinal tract & & $16(8.9)$ & & \\
\hline$\geq 3$ sepsis foci & & $5(2.8)$ & & \\
\hline Other & & $18(10.0)$ & & \\
\hline Unknown & & $66(36.7)$ & & \\
\hline \multicolumn{5}{|c|}{ Laboratory and physiological data ${ }^{\mathrm{a}}$, median (IQR) } \\
\hline White blood cell count $\left(\times 10^{9} / \mathrm{L}\right)$ & $0.90(0.09-6.23)$ & $0.60(0.06-6.39)$ & $1.08(0.14-5.8)$ & 0.093 \\
\hline Platelet count $\left(\times 10^{9} / \mathrm{L}\right)$ & $31.0(15-78)$ & $26.5(13-65)$ & $35.0(16-91)$ & 0.002 \\
\hline Creatinine $(\mu \mathrm{mol} / \mathrm{L})$ & $89.3(62.8-136.1)$ & $103.4(67.2-217.5)$ & $84.0(61.9-116.7)$ & $<0.001$ \\
\hline Bilirubin ( $\mu \mathrm{mol} / \mathrm{L})$ & $13.7(8.6-24.0)$ & $17.1(8.6-46.2)$ & $12.0(6.8-18.8)$ & $<0.001$ \\
\hline MAP $(\mathrm{mmHg})$ & $80(70-90)$ & $73(65-87)$ & $83(74-91)$ & $<0.001$ \\
\hline Temperature $\left({ }^{\circ} \mathrm{C}\right)$ & $38.1(37.2-38.5)$ & $38.2(37.0-38.7)$ & $38.1(37.6-38.4)$ & 0.482 \\
\hline Systolic pressure (mmHg) & $120(100-130)$ & $110(90-125)$ & $120(106-132)$ & $<0.001$ \\
\hline Heart rate (bpm) & $100(84-116)$ & $108(88-128)$ & $96(84-106)$ & $<0.001$ \\
\hline Lactate (mmol/L), ICU only ${ }^{\mathrm{b}}$ & $1.9(1.16-3.1)$ & $2.0(1.21-3.7)$ & $1.84(1.1-2.7)$ & 0.226 \\
\hline
\end{tabular}

Data are presented as absolute number (\%), unless otherwise indicated

$p$ value between the respective variable and sepsis with a significance level of $p$ value $<0.05$, significant differences are shown in italics

$I Q R$ interquartile range, MAP mean arterial pressure

${ }^{\mathrm{a}}$ At least $98 \%$ of the cohort contributed to the presented data

${ }^{\mathrm{b}}$ At least $90 \%$ of the cohort contributed to the presented data 
Table 2 Outcomes and severity indicators

\begin{tabular}{|c|c|c|c|c|}
\hline Variable & Total cohort $(n=450)$ & Patients with sepsis $(n=180)$ & Patients without sepsis $(n=270)$ & $p$ value \\
\hline \multicolumn{5}{|l|}{ Severity Indicators } \\
\hline Microbes detected ${ }^{\mathrm{a}}$ & $202(45.5)$ & $112(62.9)$ & $90(33.8)$ & $<0.001$ \\
\hline Acute kidney failure $^{c}$ & $82(22.2)$ & $46(31.5)$ & $36(16.1)$ & $<0.001$ \\
\hline Tumor lysis syndrome ${ }^{c}$ & $6(1.6)$ & $3(2.1)$ & $3(1.4)$ & 0.590 \\
\hline Graft-versus-host disease $^{c}$ & $20(5.4)$ & $7(4.9)$ & $13(5.8)$ & 0.708 \\
\hline Glasgow Coma Scale ${ }^{d}$, mean (SD) & $14.0(2.9)$ & $13.3(3.6)$ & $14.4(2.2)$ & $<0.001$ \\
\hline \multicolumn{5}{|l|}{ Outcomes } \\
\hline Overall in-hospital mortality & $109(24.3)$ & $71(39.7)$ & $38(14.1)$ & $<0.001$ \\
\hline ICU mortality & $80(44.4)$ & $52(51.5)$ & $28(35.0)$ & 0.023 \\
\hline Length of stay ${ }^{\mathrm{e}}$, median days (IQR) & $26(16-41)$ & $28(15-44)$ & $25.5(17-40)$ & 0.554 \\
\hline Transfer from regular ward to ICU & $49(18.3)$ & $27(34.2)$ & $22(11.6)$ & 0.005 \\
\hline \multicolumn{5}{|l|}{ Sepsis scores ${ }^{f}$} \\
\hline $\mathrm{SIRS} \geq 2$ & $351(80.1), n=438$ & $150(85.7), n=175$ & $201(76.4), n=263$ & 0.017 \\
\hline SOFA $\geq 2$ & $173(42.2), n=410$ & $112(64.0), n=175$ & $61(26.0), n=235$ & $<0.001$ \\
\hline $\mathrm{qSOFA} \geq 2$ & $90(22.0), n=410$ & $68(41.5), n=164$ & $22(8.9), n=246$ & $<0.001$ \\
\hline Septic shock & $20(4.5), n=441$ & $20(11.7), n=171$ & - & $<0.001$ \\
\hline $\operatorname{SIRS}<2$ & $3(15.0)$ & $3(15.0)$ & & \\
\hline SOFA $<2$ & $2(10.0)$ & $2(10.0)$ & & \\
\hline qSOFA $<2$ & $4(20.0)$ & $4(20.0)$ & & \\
\hline \multicolumn{5}{|l|}{ ICU $(n=181)$} \\
\hline \multicolumn{5}{|l|}{ Severity and septic shock indicators } \\
\hline APACHE $\|^{d}$, mean $(S D)$ & $22.3(8.0)$ & $24.1(8.0)$ & $20(7.5)$ & $<0.001$ \\
\hline Hypotension (MAP $<65$ mmHg) & $46(25.8)$ & $33(33.3)$ & $13(16.5)$ & $<0.001$ \\
\hline Use of vasopressors ${ }^{a}$ & $97(54.2)$ & $65(65.6)$ & $32(40.0)$ & $<0.001$ \\
\hline Lactate $>2 \mathrm{mmol} / \mathrm{L}^{\mathrm{b}}$ & $78(47.0)$ & $46(48.9)$ & $32(44.4)$ & 0.226 \\
\hline \multicolumn{5}{|c|}{ 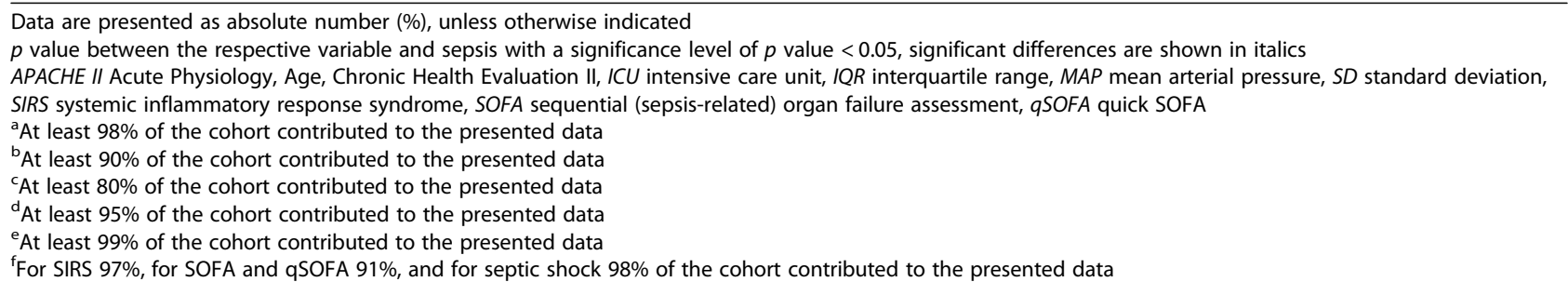 } \\
\hline
\end{tabular}

Prognostic performances of the three investigated sepsis criteria for the total cohort are reported in Table 3. The Youden's Index as an indicator for the performance of dichotomous diagnostic tests was 0.093 for SIRS, 0.380 for SOFA, and 0.325 for qSOFA. The AUROC values for discrimination of sepsis were 0.57 (95\% confidence interval $[95 \% \mathrm{CI}], 0.53-0.61 ; p<0.001)$ for SIRS, 0.69 (95\% CI, $0.64-0.73 ; p<0.001$ ) for SOFA, and 0.67 (95\% CI, 0.62-0.71; $p<0.001$ ) for qSOFA (Fig. 1). When comparing the ROC curves of SOFA with qSOFA, the areas are not significantly different $(p$ value $=0.309)$; however, comparing the ROC curves of SOFA or qSOFA with SIRS, respectively, the areas are significantly different (both $p$ values $<0.001$ ).

\section{In-hospital mortality}

For the prediction of in-hospital mortality, SIRS had a sensitivity of $79 \%(95 \% \mathrm{CI}, 71-86 \%)$ and a specificity of $20 \%$ (95\% CI, $16-24 \%$ ), SOFA had a sensitivity of $76 \%(95 \% \mathrm{CI}, 67-83 \%)$ and a specificity of $70 \%(95 \%$ CI, $64-74 \%$ ), and for qSOFA, the sensitivity was $45 \%$ (95\% CI, 36-55\%) and the specificity was $86 \%(95 \%$ CI, 81-89\%). The positive likelihood ratio was 3.1 (95\% CI, 2.2-4.4) for qSOFA and 2.5 (95\% CI, 2.13.1 ) for SOFA. The negative predictive value was $83 \%$ for $\mathrm{qSOFA}$ and $90 \%$ for SOFA, respectively. Figure 1 and Table 3 show the AUROCs and values for the prediction of in-hospital mortality by the three sepsis scores. The highest AUROCs were determined for 
Table 3 Performance of SIRS, SOFA and qSOFA scores

\begin{tabular}{|c|c|c|c|}
\hline Diagnostic accuracy and outcome measures & SIRS & SOFA & qSOFA \\
\hline \multicolumn{4}{|l|}{ Diagnosis of sepsis } \\
\hline \multicolumn{4}{|l|}{ Total cohort $(n=450)^{f}$} \\
\hline Sensitivity, \% (95\% Cl) & $85.7(79.8-90.1)$ & $64.0(56.7-70.7)$ & $41.5(34.2-49.1)$ \\
\hline Specificity, \% (95\% Cl) & $23.6(18.9-29.1)$ & $74.0(68.1-79.2)$ & $91.1(86.8-94.0)$ \\
\hline Youden's Index & 0.093 & 0.380 & 0.325 \\
\hline PPV, \% (95\% Cl) & $42.7(37.5-48.1)$ & $64.7(57.1-71.7)$ & $75.6(65.2-83.7)$ \\
\hline NPV, \% (95\% Cl) & $71.3(60.4-80.2)$ & $73.4(67.2-78.8)$ & $70.0(64.6-74.9)$ \\
\hline AUROC $(95 \% \mathrm{Cl})$ & $0.57(0.53-0.61) ; p<0.001$ & $0.69(0.64-0.73) ; p<0.001$ & $0.67(0.62-0.71) ; p<0.001$ \\
\hline \multicolumn{4}{|l|}{ In-hospital mortality } \\
\hline \multicolumn{4}{|l|}{ Total cohort $(n=450)^{f}$} \\
\hline Sensitivity, \% (95\% Cl) & $79.2(70.6-85.9)$ & $76.2(67.2-83.3)$ & $45.0(35.6-54.8)$ \\
\hline Specificity, \% (95\% Cl) & $19.6(15.7-24.2)$ & $69.5(64.1-74.4)$ & $85.5(81.1-89.0)$ \\
\hline Youden's Index & - & 0.456 & 0.304 \\
\hline AUROC $(95 \% \mathrm{Cl})$ & $0.49(0.44-0.54) ; p<0.001$ & $0.74(0.69-0.79) ; p<0.001$ & $0.65(0.60-0.71) ; p<0.001$ \\
\hline Mortality if score $\geq 2$ & $84(23.9), n=351$ & $80(46.2), n=173$ & $45(50.0), n=90$ \\
\hline Mortality if score $<2$ & $22(25.3), n=87$ & $25(10.5), n=237$ & $55(17.2), n=320$ \\
\hline Risk ratio $(95 \% \mathrm{Cl})$ & $0.95(0.63-1.42) ; p=0.792$ & $4.38(2.93-6.57) ; p<0.001$ & $2.91(2.12-3.99) ; p<0.001$ \\
\hline \multicolumn{4}{|l|}{ Patients with sepsis $(n=180)$} \\
\hline Mortality if score $\geq 2$ & $58(38.7), n=150$ & $63(56.3), n=112$ & $33(48.5), n=68$ \\
\hline Mortality if score $<2$ & $10(40.0), n=25$ & $7(11.1), n=63$ & $32(33.3), n=96$ \\
\hline Risk ratio $(95 \% \mathrm{Cl})$ & $0.97(0.57-1.63) ; p=0.899$ & $5.06(2.47-10.37) ; p<0.001$ & $1.44(0.99-2.09) ; p=0.056$ \\
\hline \multicolumn{4}{|l|}{ Patients without sepsis $(n=270)$} \\
\hline Mortality if score $\geq 2$ & $26(12.9), n=201$ & $17(27.9), n=61$ & $12(54.5), n=22$ \\
\hline Mortality if score $<2$ & $12(19.4), n=62$ & $18(10.3), n=174$ & $23(10.3), n=224$ \\
\hline Risk ratio $(95 \% \mathrm{Cl})$ & $0.67(0.36-1.24) p=0.209$ & $2.69(1.49-4.89) ; p=0.001$ & $5.31(3.08-9.15) ; p<0.001$ \\
\hline \multicolumn{4}{|l|}{ ICU patients with sepsis $(n=101)$} \\
\hline Mortality if score $\geq 2$ & $41(50.0), n=82$ & $48(56.5), n=85$ & $28(57.1), n=49$ \\
\hline Mortality if score $<2$ & $10(58.8), n=17$ & $3(25.0), n=12$ & $23(48.9), n=47$ \\
\hline Risk ratio $(95 \% \mathrm{Cl})$ & $0.85(0.54-1.34) ; p=0.508$ & $2.26(0.83-6.13) ; p=0.041$ & $1.17(0.80-1.71) ; p=0.421$ \\
\hline
\end{tabular}

fFor SIRS 97\%, for SOFA and qSOFA 91\%, and for septic shock $98 \%$ of the hematological cohort contributed to the presented data

$A U R O C$ area under receiver operating characteristic, $C l$ confidence interval, $I C U$ intensive care unit, NPV negative predictive value, $P P V$ positive predictive value, SIRS systemic inflammatory response syndrome, SOFA sequential (sepsis-related) organ failure assessment, qSOFA quick SOFA

SOFA (0.74 [95\% CI, 0.69-0.79]; $p<0.001)$ and qSOFA score $(0.65$ [95\% CI, $0.60-0.71] ; p<0.001)$ compared to SIRS $(0.49$ [95\% CI, 0.44-0.54]; $p<$ 0.001). When comparing the ROC curves of SOFA with qSOFA regarding prediction of in-hospital mortality, there was a significant difference ( $p$ value $=0.005$ ).

In the whole cohort, including ICU and non-ICU patients, in-hospital mortality was $24 \%$ with an ICU mortality of $44 \%$. Of all patients with sepsis, $40 \%$ died compared to $14 \%$ of all patients without sepsis $(p<0.001$; Table 2).

Focusing on patients with sepsis, the mortality rate in patients with positive and negative SIRS scores was 39\% vs. $40 \%(p=0.899)$, respectively. For patients with a qSOFA score $\geq 2$, mortality was $49 \%$ compared to $33 \%$ for those with a qSOFA score $<2(p=0.056)$, and for the SOFA score $56 \%$ vs. $11 \%(p<0.001)$, respectively. Thus, the mortality rate for sepsis patients with falsely negative scores was lowest (11\%) for the SOFA score. Patients with sepsis with SOFA scores $\geq 2$ were about five times more likely to die (risk ratio 5.06 [95\% CI, 2.47-10.37]) compared to patients with SOFA scores $<2$. Regarding qSOFA, patients with a qSOFA score $\geq 2$ were about 1.5 times more likely to die compared to patients with a score < 2 (risk ratio 1.44 [95\% CI, 0.99-2.09]). In contrast, there was little difference in mortality in patients with $\geq 2$ SIRS scores compared to patients with SIRS scores $<2$ (risk ratio 0.97 [95\% CI, 0.57-1.63]). In the group of patients with sepsis treated on the ICU, patients with $\geq 2$ SOFA scores were more than twice as 

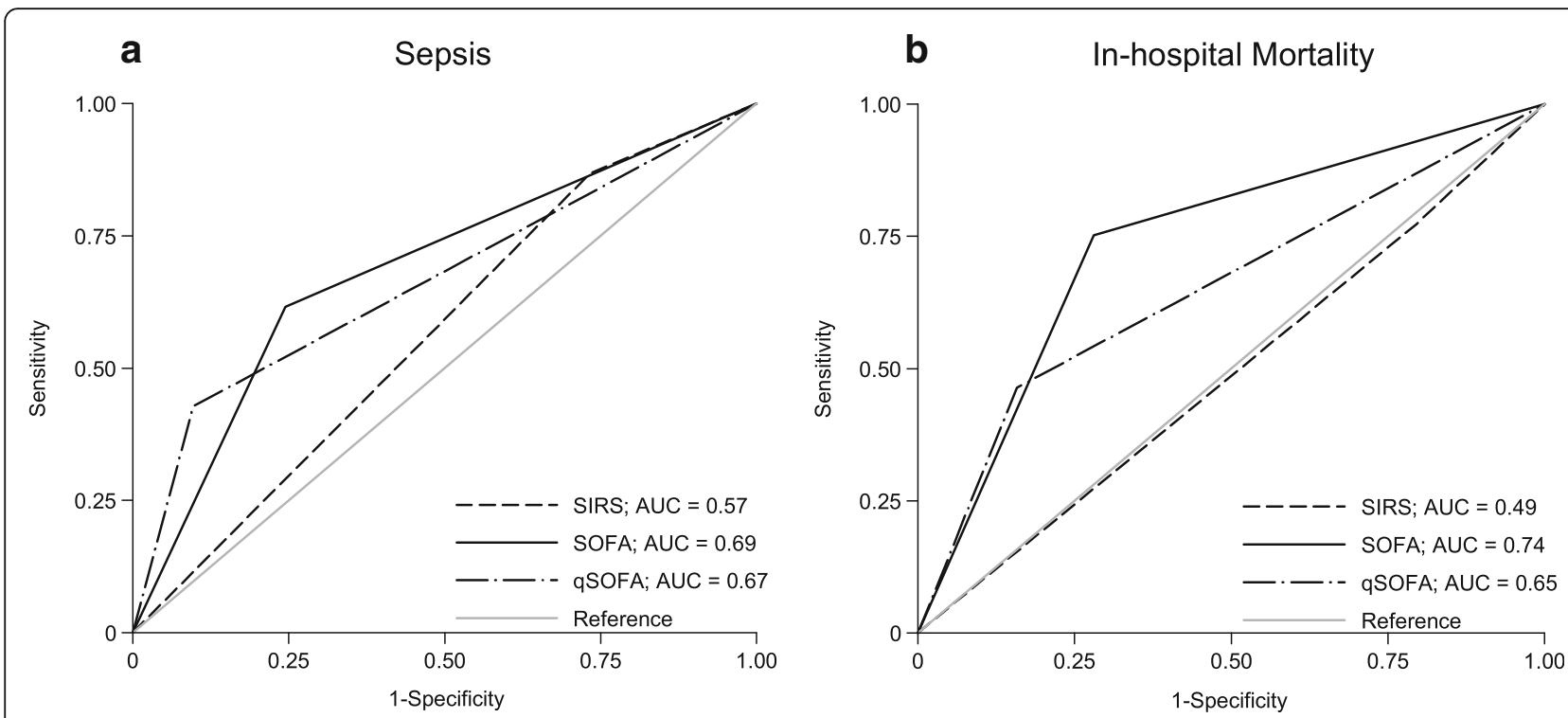

Fig. 1 Receiver operating characteristic (ROC) curves (unadjusted) for a sepsis and $\mathbf{b}$ in-hospital mortality. The areas under the ROC curves (AUROCS) for panel a are SIRS, 0.57 (95\% confidence interval [CI], 0.53-0.61), $p<0.001$; SOFA, 0.69 (95\% Cl, 0.64-0.73), $p<0.001$; qSOFA, 0.67 (95\% $\mathrm{Cl}, 0.62-0.71), p<0.001$, and for panel b SIRS, 0.49 (95\% Cl, 0.44-0.54), $p<0.001$; SOFA, 0.74 (95\% Cl, 0.69-0.79), $p<0.001$; qSOFA, 0.65 (95\% Cl, $0.60-0.71), p<0.001$. AUC area under the curve, SIRS systemic inflammatory response syndrome, SOFA sequential (sepsis-related) organ failure assessment, qSOFA quick SOFA

likely to die as patients with SOFA scores $<2$ (risk ratio 2.26 [95\% CI, 0.83-6.13]), being the only risk ratio with a significance level $<0.05$ in this group (Table 3).

\section{Discussion}

As population-based studies show, about one in five patients in the ICU has cancer and the proportion of ICU patients suffering from cancer is expected to increase over the next decades $[18,21,31]$. Patients with hematological malignancies are at particular risk of requiring ICU treatment as therapy- or disease-related immunodeficiency is common in hematological cancer patients frequently leading to infection and sepsis $[20,31]$. At the same time, patients with neutropenia and immunodeficiency frequently do not exhibit fever, pus, and other symptoms associated with the infection; thus, especially in patients with hematological malignancies and sepsis, it can be difficult to determine the site of infection (37\%). In addition, many patients suffer from polymicrobial infection originating from mucosal barrier injury, which is difficult to diagnose with conventional methods and criteria.

Although the accurate definition and early diagnosis of sepsis indisputably improve patient survival, there is a considerable debate about new definitions and criteria for sepsis [2-13, 15-17]. Moreover, the discriminatory capacity of different criteria and scores, such as SIRS, SOFA, and qSOFA, for identifying sepsis and predicting in-hospital mortality in hematological cancer patients remains unclear.
The focus of our study was the evaluation of the diagnostic validity of these criteria for the diagnosis of sepsis and the prediction of high risk of in-hospital mortality in a multicentric population of hematological cancer patients. We studied the criteria in analogy to the Sepsis-III study focusing on the criteria and their (bedside) validity, rather than on changes of inflammation markers, which has been studied previously by several groups [32, 33].

In our study, we found a higher non-ICU mortality of $11 \%$ compared to the mortality of $3 \%$ reported in the original Sepsis-3 study from Seymour and colleagues in a general population [7]. ICU mortality of hematological cancer patients in our study was $44 \%$ and even increased up to $50 \%$ if patients suffered from sepsis, which is within the reported range for hematological cancer ICU patients of 34-68\% [34] and slightly higher than reported for the largest published hematological cancer cohort by Azoulay et al. (39\%) [20]. Costa et al. studied a single-center cohort of 450 cancer patients admitted to the ICU, including about $19 \%$ of patients with hematological malignancies, and reported an overall mortality of 39\% [17]. Soares and colleagues studied a very large multicenter retrospective cohort of 9946 cancer patients, including about $10 \%(n=990)$ of patients with hematologic malignancies, reporting ICU and inhospital mortality rates of $16 \%$ and $25 \%$, respectively [21]. These findings are well in line with previous reports, indicating a higher mortality of hematological cancer ICU patients compared to studies including patients with solid tumors, which often include a higher 
proportion of patients with post-surgical surveillance $[20,21,31]$. Neutropenia, which is common in hematological cancer patients, but less in patients with solid tumors, might be an important factor. Although earlier data were conflicting, neutropenia was independently associated with an increased mortality in a recent meta-analysis including 4149 (55\%) patients with hematological cancer [35], which might contribute to the high mortality we observed.

Regarding the validity and performance of different sepsis scores in our cohort, we found that more than one in six patients $(17 \%)$ with sepsis did not meet two or more SIRS criteria. This proportion of "SIRSnegative" sepsis patients in our study was slightly higher than previously reported by Kaukonen et al. (12\%) in a population-based ICU cohort [36]. Costa et al. reported exclusively on cancer patients with a suspected infection admitted to the ICU and observed about one fifth of patients with sepsis who met less than two SIRS criteria (23\%) [17]. However, both studies did not include information on the respective criteria in patients without sepsis $[17,36]$. Interestingly, the majority of patients in our study without sepsis met two or more SIRS criteria (76\%). In those false-positive patients, almost all patients $(90 \%)$ had abnormal white blood cell counts $\left(<4 \times 10^{9} / \mathrm{L}\right.$ or $>$ $12 \times 10^{9} / \mathrm{L}$ ), which were responsible for most positive SIRS scores in patients without sepsis. This observation of a high proportion of "SIRS-positive" patients without sepsis was similarly reported by several authors in different populations including ICU and non-ICU patients [13]. Therefore SIRS criteria are of limited use in diagnosing and screening for sepsis in hematological cancer patients as abnormal white blood cell counts are a common disease- or therapyrelated observation in these populations.

Both scores, SOFA and qSOFA, had a lower proportion of false-positive patients; however, the proportion of patients with sepsis, who did not meet two or more of the criteria, also increased. Thirty-six percent of the patients with sepsis did not meet a positive SOFA score (two or more) and more than half of the patients with sepsis (59\%) had less than two of three qSOFA criteria (Table 3). The qSOFA was mainly negative due to a GCS of 15 (in $92 \%$ of the patients) and a systolic blood pressure above $100 \mathrm{mmHg}$ ( $73 \%$ of the patients).

Yet, the mortality rate for the $36 \%$ of sepsis patients with false-negative SOFA scores was low (11\%) compared to the respective mortality rates for SIRS (40\%) and qSOFA (33\%).

Overall, we found that SOFA was more accurate than qSOFA or SIRS in discriminating hematological cancer patients with sepsis from patients without sepsis (SOFA, AUROC 0.69 [95\% CI, 0.64-0.73], $p<0.001$; qSOFA,
AUROC 0.67 [95\% CI, 0.62-0.71], $p<0.001 ;$ SIRS, AUROC 0.57 [95\% CI, 0.53-0.61], $p<0.001$; Table 3). Focusing on ICU patients, the sensitivity in hematological cancer patients was highest for SOFA (88\%) and the false-negative rate for ICU patients with sepsis was lower (12\%) compared to the other two scoring systems.

Moreover, in-hospital mortality in hematological cancer patients with sepsis was about fivefold higher in "SOFA-positive" patients compared to patients with a SOFA score below two (risk ratio 5.06 [95\% CI, $2.47-10.37] ; p<0.001)$. The discrimination of in-hospital mortality using SOFA (AUROC 0.74 [95\% CI, $0.69-0.79$ ]; $p<0.001)$ was significantly higher compared with SIRS criteria (AUROC 0.49 [95\% CI, 0.44$0.54] ; p<0.001$ ), for which the mortality rate was $40 \%$ in "SIRS-negative" patients and about 39\% in "SIRSpositive" patients (Table 3). Similarly, several authors previously suggested SOFA as predictor of ICU mortality in general ICU populations with suspected infection, and the relationship between SOFA and mortality has been investigated in a variety of subgroups [7, 17, 37-40].

Importantly, there are significant limitations to the clinical use of the SOFA score, as it requires laboratory measurements and is time-consuming to calculate. However, our results in a hematological cancer population including non-ICU patients suggest that SIRS criteria are not accurate in hematological cancer patients and that also qSOFA, which has been proposed as an effective way of raising suspicion of sepsis on the regular hematology ward might not be applicable in hematological cancer patients, as the number of qSOFAnegative sepsis patients with high mortality was considerable in our study.

Strengths of our study are the large number of hematological cancer patients and the multicentric design including patients from three tertiary academic hospitals. Furthermore, our cohort included a broad range of hematological cancer patients regarding disease status, HSCT, and organ dysfunction. The main limitation of the study was the retrospective study design with data acquisition possibly being prone to bias. In addition, some of the data needed to calculate the SOFA score could not be collected for non-ICU patients. Furthermore, despite the fact that we found a strong association of several characteristics with mortality including indicators of organ failure, we could not determine the impact of each single factor in a multivariate analysis, as the heterogeneity of our study population was too high regarding the sample size. A multivariate analysis would require a much higher number of patients to provide reproducible results and was not a focus of our study. 


\section{Conclusions}

In conclusion, we found that in our cohort of patients with hematological malignancies, an increase in SOFA score of $\geq 2$ had superior prognostic accuracy for discriminating sepsis and estimating in-hospital mortality compared with qSOFA or SIRS criteria. Especially on intensive care, we observed limited validity of SIRS and qSOFA in identifying hematological patients with sepsis and at high risk of death. The significant proportion of sepsis patients with a negative score, irrespective of the scoring system used, underlines the need for a clinical diagnosis of sepsis particularly in hematological cancer patients.

\section{Additional files}

Additional file 1: $2 \times 2$ contingency tables for a SIRS criteria, b SOFA score and c qSOFA score. (DOCX $23 \mathrm{~kb})$

Additional file 2: Flow charts for a SIRS criteria, b SOFA score and $c$ qSOFA score. (PPTX $44 \mathrm{~kb}$ )

\section{Abbreviations}

APACHE II: Acute Physiology, Age, Chronic Health Evaluation II; AUROC: Area under receiver operating characteristic; Cl: Confidence interval; GCS: Glasgow coma scale; HSCT: Hematopoietic stem cell transplant; ICU: Intensive care unit; IQR: Interquartile range; LOS: Length of stay; LR: Likelihood ratio; MAP: Mean arterial pressure; qSOFA: Quick sequential (sepsis-related) organ failure assessment; ROC: Receiver operating characteristic; RR: Risk ratio; SD: Standard deviation; SIRS: Systemic inflammatory response syndrome; SOFA: Sequential (sepsis-related) organ failure assessment

\section{Acknowledgements}

Not applicable.

\section{Authors' contributions}

BB and LP planned and designed the study, statistically analyzed and interpreted the data, and drafted the manuscript. All authors contributed to the acquisition of data and critically revised and approved the final version of the manuscript.

\section{Funding}

This study was not funded.

\section{Availability of data and materials}

The datasets used and/or analyzed during the current study are available from the corresponding author on reasonable request.

\section{Ethics approval and consent to participate}

The Institutional Ethical Review Board of the study center (Medical Faculty of the University of Cologne), approved the study and waved the need for informed consent due to the non-interventional retrospective design of the investigation.

\section{Consent for publication}

Not applicable.

\section{Competing interests}

The authors declare that they have no competing interests.

\section{Author details}

${ }^{1}$ University of Cologne, Department I of Internal Medicine, Center for Integrated Oncology Aachen Bonn Cologne Duesseldorf, Cologne, Germany. ${ }^{2}$ Department of Hematology and Oncology, Otto-von-Guericke University, Magdeburg, Germany. ${ }^{3}$ Department of Bone Marrow Transplantation, West German Cancer Center, University Hospital Essen, University of
Duisburg-Essen, Essen, Germany. ${ }^{4}$ Department of Medicine III, University Hospital LMU Munich, Munich, Germany. ${ }^{5}$ German Centre for Infection Research, partner-site Bonn-Cologne, Cologne, Germany. ${ }^{6}$ Medical Department 2, Hematology/Oncology, Goethe University of Frankfurt, Frankfurt, Germany.

Received: 11 May 2019 Accepted: 24 July 2019

Published online: 07 August 2019

\section{References}

1. Fleischmann C, Scherag A, Adhikari NKJ, Hartog CS, Tsaganos T, Schlattmann P, et al. Assessment of global incidence and mortality of hospital-treated Sepsis. Current estimates and limitations. Am J Respir Crit Care Med. 2015;193(3):259-72.

2. Rhodes A, Evans LE, Alhazzani W, Levy MM, Antonelli M, Ferrer R, et al. Surviving sepsis campaign: international guidelines for management of sepsis and septic shock: 2016. Crit Care Med. 2017;45(3):486-552.

3. Shankar-Hari M, Phillips GS, Levy ML, Seymour CW, Liu VX, Deutschman CS, et al. Developing a new definition and assessing new clinical criteria for septic shock: for the third international consensus definitions for sepsis and septic shock (Sepsis-3). JAMA. 2016;315(8):775-87.

4. Cohen J, Vincent JL, Adhikari NKJ, et al. Sepsis: a roadmap for future research. Lancet Infect Dis. 2015;15:581-614.

5. Levy MM, Evans LE, Rhodes A. The surviving Sepsis campaign bundle: 2018 update. Crit Care Med. 2018;46(6):997-1000.

6. Singer M, Deutschman CS, Seymour CW, Shankar-Hari M, Annane D, Bauer $M$, et al. The third international consensus definitions for sepsis and septic shock (Sepsis-3). JAMA. 2016;315(8):801-10.

7. Seymour CW, Liu VX, Iwashyna TJ, Brunkhorst FM, Rea TD, Scherag A, et al. Assessment of clinical criteria for sepsis: for the third international consensus definitions for sepsis and septic shock (Sepsis-3). JAMA. 2016; 315(8):762-74.

8. Finkelsztein EJ, Jones DS, Ma KC, et al. Comparison of qSOFA and SIRS for predicting adverse outcomes of patients with suspicion of sepsis outside the intensive care unit. Crit Care. 2017;21:73.

9. IDSA Sepsis Task Force. Infectious Diseases Society of America (IDSA) POSITION STATEMENT: why IDSA did not endorse the surviving Sepsis campaign guidelines. Clin Infect Dis. 2018;66:1631-5.

10. Simpson SQ. SIRS in the time of Sepsis-3. Chest. 2018;153(1):34-8.

11. Carneiro AH, Póvoa P, Gomes JA. Dear Sepsis-3, we are sorry to say that we don't like you. Rev Bras Ter Intensiva. 2017;29(1):4-8.

12. Simpson SQ. New Sepsis criteria: a change we should not make. Chest. 2016;149(5):1117-8

13. Vincent JL, Martin GS, Levy MM. qSOFA does not replace SIRS in the definition of sepsis. Crit Care. 2016;20:210.

14. Kim M, Ahn S, Kim WY, Sohn CH, Seo DW, Lee Y-S, et al. Predictive performance of the quick sequential organ failure assessment score as a screening tool for sepsis, mortality, and intensive care unit admission in patients with febrile neutropenia. Support Care Cancer. 2017;25(5):1557-62.

15. Tridente A. Sepsis 3 and the burns patient: do we need Sepsis 3.1? Scars Burns Heal. 2018:4:2059513118790658

16. Sartelli M, Kluger Y, Ansaloni L, Hardcastle TC, Rello J, Watkins RR, et al. Raising concerns about the Sepsis-3 definitions. World J Emerg Surg WJES. 2018;13:6.

17. Costa RT, Nassar AP, Caruso P. Accuracy of SOFA, qSOFA, and SIRS scores for mortality in cancer patients admitted to an intensive care unit with suspected infection. J Crit Care. 2018:45:52-7.

18. Taccone FS, Artigas AA, Sprung CL, et al. Characteristics and outcomes of cancer patients in European ICUs. Crit Care. 2009;13:R15.

19. Azoulay $E$, Pène $F$, Darmon $M$, Lengliné $E$, Benoit $D$, Soares $M$, et al. Managing critically ill hematology patients: time to think differently. Blood Rev. 2015;29(6):359-67.

20. Azoulay $E$, Mokart $D$, Pène $F$, et al. Outcomes of critically ill patients with hematologic malignancies: prospective multicenter data from France and Belgium--a groupe de recherche respiratoire en réanimation oncohématologique study. J Clin Oncol. 2013;31:2810-8.

21. Soares M, Bozza FA, Azevedo LCP, Silva UVA, Corrêa TD, Colombari F, et al. Effects of organizational characteristics on outcomes and resource use in patients with cancer admitted to intensive care units. J Clin Oncol. 2016; 34(27):3315-24. 
22. Rosolem MM, Rabello LSCF, Lisboa T, Caruso P, Costa RT, Leal JVR, et al. Critically ill patients with cancer and sepsis: clinical course and prognostic factors. J Crit Care. 2012;27(3):301-7.

23. American College of Chest Physicians/Society of Critical Care Medicine Consensus Conference: Definitions for sepsis and organ failure and guidelines for the use of innovative therapies in sepsis. Crit Care Med. 1992; 20(6):864-874

24. Knaus WA, Draper EA, Wagner DP, Zimmerman JE. APACHE II: a severity of disease classification system. Crit Care Med. 1985 Oct;13(10):818-29.

25. Teasdale G, Jennett B. Assessment of coma and impaired consciousness. a practical scale. Lancet. 1974;2:81-4.

26. Bone RC, Balk RA, Cerra FB, Dellinger RP, Fein AM, Knaus WA, et al. Definitions for sepsis and organ failure and guidelines for the use of innovative therapies in Sepsis. Chest. 1992;101(6):1644-55.

27. Hilden J, Glasziou P. Regret graphs, diagnostic uncertainty and Youden's index. Stat Med. 1996;15(10):969-86.

28. Youden WJ. Index for rating diagnostic tests. Cancer. 1950;3(1):32-5.

29. Biggerstaff BJ. Comparing diagnostic tests: a simple graphic using likelihood ratios. Stat Med. 2000;19(5):649-63.

30. McGee S. Simplifying likelihood ratios. J Gen Intern Med. 2002 Aug; 17(8):647-50.

31. Shimabukuro-Vornhagen A, Böll B, Kochanek M, Azoulay É, von BergweltBaildon MS. Critical care of patients with cancer. CA Cancer J Clin. 2016; 66(6):496-517.

32. Ebihara Y, Kobayashi K, Ishida A, Maeda T, Takahashi N, Taji Y, et al. Diagnostic performance of procalcitonin, presepsin, and C-reactive protein in patients with hematological malignancies. J Clin Lab Anal. 2017;31:6.

33. Stoma I, Karpov I, Uss A, Rummo O, Milanovich N, Iskrov I. Diagnostic value of sepsis biomarkers in hematopoietic stem cell transplant recipients in a condition of high prevalence of gram-negative pathogens. Hematol Oncol Stem Cell Ther. 2017;10(1):15-21.

34. Demandt AMP, Geerse DA, Janssen BJP, Winkens B, Schouten HC, van Mook WNKA. The prognostic value of a trend in modified SOFA score for patients with hematological malignancies in the intensive care unit. Eur J Haematol. 2017:99(4):315-22.

35. Georges $\mathrm{Q}$, Azoulay E, Mokart D, et al. Influence of neutropenia on mortality of critically ill cancer patients: results of a meta-analysis on individual data. Crit Care. 2018;22:326.

36. Kaukonen K-M, Bailey M, Pilcher D, Cooper DJ, Bellomo R. Systemic inflammatory response syndrome criteria in defining severe sepsis. N Engl J Med. 2015 Apr 23;372(17):1629-38.

37. Raith EP, Udy AA, Bailey M, McGloughlin S, Maclsaac C, Bellomo R, et al, Prognostic accuracy of the SOFA Score, SIRS criteria, and qSOFA score for in-hospital mortality among adults with suspected infection admitted to the intensive care unit. JAMA. 2017;317(3):290-300

38. Vincent J-L, Moreno R, Takala J, Willatts S, De Mendonça A, Bruining H, et al. The SOFA (sepsis-related organ failure assessment) score to describe organ dysfunction/failure. Intensive Care Med. 1996:22(7):707-10.

39. Freund Y, Lemachatti N, Krastinova E, Van Laer M, Claessens Y-E, Avondo A, et al. Prognostic accuracy of sepsis-3 criteria for in-hospital mortality among patients with suspected infection presenting to the emergency department. JAMA. 2017:317(3):301-8.

40. Geerse DA, Span LFR, Pinto-Sietsma S-J, van Mook WNKA. Prognosis of patients with haematological malignancies admitted to the intensive care unit: sequential organ failure assessment (SOFA) trend is a powerful predictor of mortality. Eur J Intern Med. 2011;22(1):57-61.

\section{Publisher's Note}

Springer Nature remains neutral with regard to jurisdictional claims in published maps and institutional affiliations.

Ready to submit your research? Choose BMC and benefit from:

- fast, convenient online submission

- thorough peer review by experienced researchers in your field

- rapid publication on acceptance

- support for research data, including large and complex data types

- gold Open Access which fosters wider collaboration and increased citations

- maximum visibility for your research: over $100 \mathrm{M}$ website views per year

At $\mathrm{BMC}$, research is always in progress.

Learn more biomedcentral.com/submissions 